\title{
Quantum simulation of two-dimensional quantum chemistry in optical lattices
}

\author{
Javier Argüello-Luengo $\odot,{ }^{1, *}$ Alejandro González-Tudela $\odot,{ }^{2, \dagger}$ Tao Shi, ${ }^{3, *}$ Peter Zoller,,${ }^{4,5}$ and J. Ignacio Cirac ${ }^{6,7, \S}$ \\ ${ }^{1}$ ICFO-Institut de Ciències Fotòniques, The Barcelona Institute of Science and Technology, E-08860 Castelldefels (Barcelona), Spain \\ ${ }^{2}$ Instituto de Física Fundamental IFF-CSIC, Calle Serrano 113b, E-28006 Madrid, Spain \\ ${ }^{3}$ CAS Key Laboratory of Theoretical Physics, Institute of Theoretical Physics, Chinese Academy of Sciences, P.O. Box 2735, \\ Beijing 100190, China \\ ${ }^{4}$ Center for Quantum Physics, University of Innsbruck, A-6020 Innsbruck, Austria \\ ${ }^{5}$ Institute for Quantum Optics and Quantum Information of the Austrian Academy of Sciences, Innsbruck, Austria \\ ${ }^{6}$ Max-Planck-Institut für Quantenoptik, Hans-Kopfermann-Straße 1, D-85748 Garching, Germany \\ ${ }^{7}$ Munich Center for Quantum Science and Technology (MCQST), München, Germany
}

(Received 2 March 2020; accepted 21 August 2020; published 16 October 2020)

\begin{abstract}
Benchmarking numerical methods in quantum chemistry is one of the key opportunities that quantum simulators can offer. Here, we propose an analog simulator for discrete two-dimensional quantum chemistry models based on cold atoms in optical lattices. We first analyze how to simulate simple models, such as the discrete versions of $\mathrm{H}$ and $\mathrm{H}_{2}{ }^{+}$, using a single fermionic atom. We then show that a single bosonic atom can mediate an effective Coulomb repulsion between two fermions, leading to the analog of molecular hydrogen in two dimensions. We extend this approach to larger systems by introducing as many mediating atoms as fermions, and derive the effective repulsion law. In all cases, we analyze how the continuous limit is approached for increasing optical lattice sizes.
\end{abstract}

DOI: 10.1103/PhysRevResearch.2.042013

The field of theoretical quantum chemistry has experienced extraordinary progress due, in part, to many advances in computational methods [1]. For instance, density functional theory (DFT) $[2,3]$ has enabled a better description and understanding of both static [4-7] and dynamic [8] properties of a large variety of molecules. The capability of such computational methods, whose main challenge is to address electronic correlations, is however sometimes hard to assess experimentally. One approach is to use another (classical) computational technique that is exact in some restricted conditions, but can deal with large systems where exact calculations were not possible. The most prominent example is density matrix renormalization group (DMRG) [9] which, despite the fact that it operates in one-dimensional (1D) lattice systems, offers an ideal platform to benchmark DFT methods [10-13]. In more general scenarios, the field of quantum computing [14-20] can play a key role to overcome numerical limitations in the long term, offering an excellent setup to benchmark quantum chemistry computational methods. Recently, we have proposed the alternative approach of analog quantum simulation [21], based on

\footnotetext{
*javier.arguello@icfo.eu

†a.gonzalez.tudela@csic.es

†tshi@itp.ac.cn

\$ignacio.cirac@mpq.mpg.de
}

Published by the American Physical Society under the terms of the Creative Commons Attribution 4.0 International license. Further distribution of this work must maintain attribution to the author $(s)$ and the published article's title, journal citation, and DOI. the experimentally mature field of ultracold atoms [22-24], where fermionic atoms play the role of the electrons. While quantum computers and analog simulators would certainly help to push quantum chemistry, the exploration of their full potentiality requires the development of techniques that go beyond the state of the art.

In this Rapid Communication we propose and analyze a scheme for analog quantum chemistry simulation that can be implemented with present technology. Our approach uses ultracold atoms to address lattice models in two spatial dimensions (2D), where the electron-electron interaction takes different forms. While not exactly reproducing all aspects of the real quantum chemistry scenario, this simulator still retains the most relevant ingredients, enabling the observation of the most representative phenomena in quantum chemistry. Furthermore, it offers a suitable platform to benchmark computational methods in that field. In particular, it allows us to extend the benchmarking offered by DMRG beyond 1D [25].

For the sake of clarity, we will discuss several scenarios, with increasing experimental difficulty, for the simulation of quantum chemistry problems in 2D discrete lattices that could later be compared to contemporary theoretical methods, such as DFT or tensor network methods [26,27]. We start with simple one-electron systems, analogous to the hydrogen atom, and the $\mathrm{H}_{2}{ }^{+}$molecule. Then, we show how to simulate two-electron problems, here exemplified by the $\mathrm{H}_{2}$ molecule. Finally, we show how the system can be scaled up to more electrons, although with a different dependence of the repulsion with the distance.

Model. In the following, we will consider a discrete version of quantum chemistry models in 2D. First, we start by 
(a)

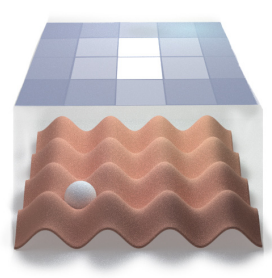

(b)
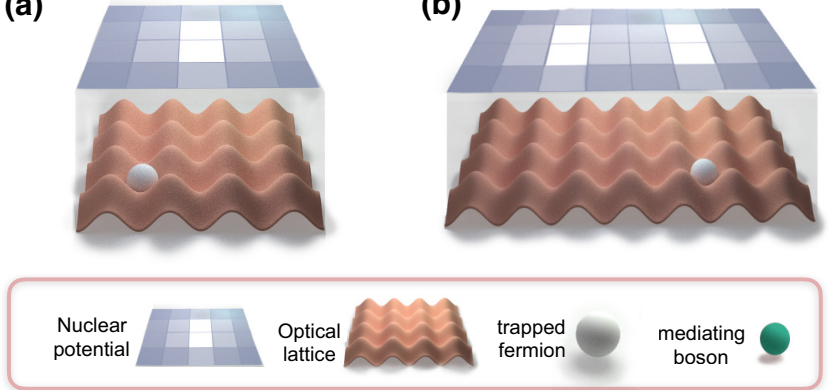

(c)

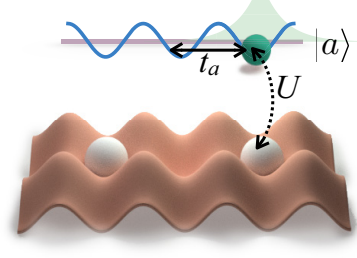

(d)

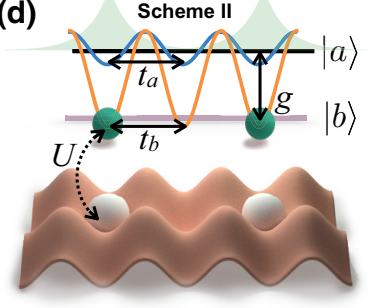

FIG. 1. Fermionic atoms (white) play the role of the molecular electrons. They hop in a 2D lattice (red), where the nuclear potential is imprinted (blue). For a single simulated electron, this pattern can lead to, e.g., atomic hydrogen [(a), one nucleus] or $\mathrm{H}_{2}{ }^{+}$[(b), two nuclei]. For more than one fermionic atom, two different schemes are proposed to mediate an effective repulsion between them. (c) A single atom (green) is used. It tunnels with constant $t_{a}$ through a lattice with the same spacing as the fermionic one. There is an on-site repulsion with strength $U$ when the mediating atom occupies the same site as the fermion. (d) We use as many mediating atoms as electrons need to be simulated (two in the case of the figure). The on-site repulsion with the fermions now appears in a different internal level $b$, whose tunneling is slower as compared to level $a$, using a state-dependent lattice. Both levels are coherently coupled with coupling constant $g$.

considering a 2D square optical lattice of size $N \times N . N_{f}$ fermionic atoms, playing the role of electrons (see Fig. 1), can localize within the local minima of this optical lattice, and hop with nearest-neighbor tunneling rate $t_{F}$. The Hamiltonian describing their dynamics is then given by

$$
H_{K}=-t_{F} \sum_{\langle\mathbf{i}, \mathbf{j}\rangle} f_{\mathbf{i}}^{\dagger} f_{\mathbf{j}}
$$

where $f_{\mathbf{i}}^{\dagger}$ and $f_{\mathbf{i}}$ are the creation and annihilation operators for a fermionic atom in the ith lattice site [28], each of them separated by a lattice spacing a, and where the sum is taken over all nearest-neighbor pairs of lattice sites. Fermionic atoms are subject to an external potential that induces the attraction to $N_{\text {nuc }}$ nuclei that we consider placed in fixed positions $\left\{\mathbf{r}_{n}\right\}_{n=1 \ldots N_{\text {nuc }}}$ [29] (Born-Oppenheimer approximation [30]),

$$
H_{\mathrm{n}}\left(\left\{\mathbf{r}_{n}\right\}\right)=-\sum_{n=1}^{N_{\text {nuc }}} \sum_{\mathbf{j}} Z_{n} V\left(\left|\mathbf{j}-\mathbf{r}_{n}\right|\right) f_{\mathbf{j}}^{\dagger} f_{\mathbf{j}},
$$

where $Z_{n}$ is the atomic number of nucleus $n$, and $V(r)$ is the attractive nuclear potential [31]. In 2D lattices, this potential can be obtained by combining the light shift induced by an external laser orthogonal to the lattice and a fully programmable intensity mask using, for example, a digital mirror device
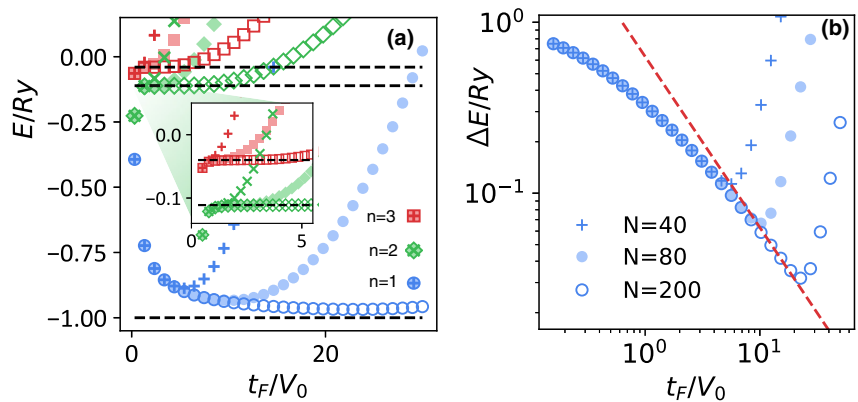

FIG. 2. (a) Lower part of the spectrum for the discretized 2D atomic hydrogen Hamiltonian in Eq. (3) for different values of the effective Bohr radius $t_{F} / V_{0}$. For clarity, only the lowest-energy state is shown for the energy levels $n=1,2,3$. As more lattice sites are involved in the simulation ( $t_{F} / V_{0}$ increases), the spectrum approaches the value in the continuum (horizontal lines for $n=1,2,3$ ). This is valid up to a critical Bohr radius in which finite-size effects become relevant and the solution deviates from this behavior. This critical value appears earlier for smaller sizes ( $N=40$ for crossed markers) than for bigger systems ( $N=80$, colored marker, and $N=200$, edged marker). (b) The energy difference $\Delta E$ between the ground state of the discretized Hamiltonian in Eq. (3), and the one in the continuum decreases polynomially before finite-size effects become relevant [36]. Larger system sizes can follow this scaling up to more precise solutions. The dashed line follows the scaling $\left(t_{F} / V_{0}\right)^{-1}$.

[32]. Depending on the model to be simulated, we will also consider the Hamiltonian $H_{\text {med }}$ describing a set of bosonic atoms that mediates fermion-fermion interactions according to some effective potential $V_{\text {eff }}$.

We consider now the simplest situation of simulating atomic hydrogen. By choosing a potential with a unique nucleus $Z_{1}=1$ centered in the lattice site $\mathbf{r}_{1}=(\lfloor N / 2\rfloor,\lfloor N / 2\rfloor+$ $1 / 2$ ), the total Hamiltonian reads as

$$
H_{1}=H_{K}+H_{n}\left(\mathbf{r}_{1}\right) \text {. }
$$

To begin with, we consider the attractive Coulomb potential on its standard form, $V(r)=V_{0} / r$, for moderate finite lattice sizes, e.g., $N=40$. In order to gain intuition, one can compare this discretized Hamiltonian to the continuum limit, where an analytical solution is also known in 2D [33]. As a consequence of the reduced dimensionality, electrons get closer to the nuclei than in the 3D case [34]. Each energy level corresponds to $E_{n}^{*}=\frac{-\mathrm{Ry}}{(n-1 / 2)^{2}}$, for $n=1,2, \ldots$. In that limit, one can also identify

$$
\mathrm{a}_{0} / \mathrm{a}=t_{F} / V_{0} \quad \text { and } \quad \mathrm{Ry}=V_{0}^{2} / t_{F},
$$

that are the equivalent Bohr radius $\left(\mathrm{a}_{0}\right)$ and Rydberg energy (Ry) for the 2D discrete model [35]. The first ultimately determines the size of the orbitals and thus how the continuum limit is recovered. In particular, it is needed that the orbitals fit in the lattice (to avoid finite-size effects), and that this Bohr radius occupies several lattice sites (to avoid discretization errors), leading to the inequalities

$$
N \gg t_{F} / V_{0} \gg 1 .
$$

In Fig. 2(a) we show the lower part of the spectrum of the discretized Hamiltonian (3) for different values of $t_{F} / V_{0}$ and $N$. First, we observe that we have quantized levels, and thus 

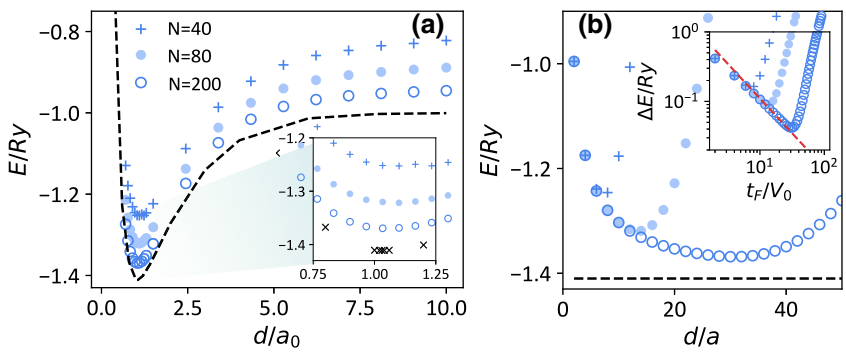

FIG. 3. (a) Ground-state energy of the 2D hydrogen cation $\left(\mathrm{H}_{2}{ }^{+}\right)$ for different lattice sizes $N$ and internuclear distance $d / \mathrm{a}_{0}$ (see text for the optimal choice of the lattice separation). The inset zooms into separation close to equilibrium. The dashed line (black crosses in the inset) follows an accurate solution for this 2D cation [37]. (b) Ground-state energy of $\mathrm{H}_{2}{ }^{+}$calculated for fixed $d / \mathrm{a}_{0}=1$ and increasing effective Bohr radius $t_{F} / V_{0}$. The energy of the calculated ground state decreases up to a critical size at which finite-size effects appear. This critical size is larger for bigger lattice sizes. In the inset, the difference in energies to the tabulated value -1.41 Ry (black dashed line) reveals the scaling $\left(t_{F} / V_{0}\right)^{-1}$ (red dashed line). Markers represent the same sizes as in (a).

the discrete model qualitatively reproduces the continuous one. In fact, this can be observed with small lattices $(N=40)$. Quantitatively, we see that by increasing the ratio $t_{F} / V_{0}$ and making the lattice larger, one approaches the continuum limit, as intuitively expected. The error for this approximation as a function of $t_{F} / V_{0}$ is shown in Fig. 2(b), where it is observed to scale approximately as $\left(t_{F} / V_{0}\right)^{-1}$ [see Supplemental Material (SM) [36]].

Let us now explore a system with a single fermion and two equal nuclei, $Z_{1,2}=1$, separated by $d /$ a lattice sites, $\mathbf{r}_{1,2}=$ $(\lfloor N / 2 \pm d /(2 \mathrm{a})\rfloor,\lfloor N / 2\rfloor+1 / 2)$, i.e., the analog of $\mathrm{H}_{2}{ }^{+}$. This internuclear separation measured in number of lattice sites can be directly expressed in terms of the Bohr radius as $d / \mathrm{a}_{0}=(d / \mathrm{a})\left(V_{0} / t_{F}\right)$, and therefore compared to tabulated values [37]. In Fig. 3(a) we plot the energy of the ground state as a function of the distance. We obtain a molecular potential, as it is expected for $\mathrm{H}_{2}{ }^{+}$, already for the moderate size $N=40$. Increasing $t_{F} / V_{0}$ favors accuracy, up to the point where finite-size effects appear. At this point the difference in energies to the continuum (dashed line) deviates from the universal scaling $\Delta E \propto\left(t_{f} / V_{0}\right)^{-1}$, which identifies the optimal configuration for our finite system and a given choice of $d / \mathrm{a}_{0}$. In Fig. 3(b) we illustrate this effect by showing that a given internuclear separation $d / \mathrm{a}_{0}$ can be calculated with different values of integer lattice-site separations $d / \mathrm{a}$ by tuning the effective Bohr radius $\mathrm{a}_{0} / \mathrm{a}$ accordingly (see Ref. [36]).

Two-fermion model. Let us now explore the situation with two fermionic atoms emulating two electrons, where the interelectronic repulsion between them needs to be mediated. For this, we use an additional bosonic atom trapped in an optical lattice potential with the same geometry as the fermions. First, we start with a simple scheme that only considers one of the bosonic internal states, which allows them to tunnel at a rate $t_{a}$ to nearest-neighboring sites. As they coexist in the same lattice sites, elastic scattering processes between the bosonic and fermionic atoms occupying the same position induce an
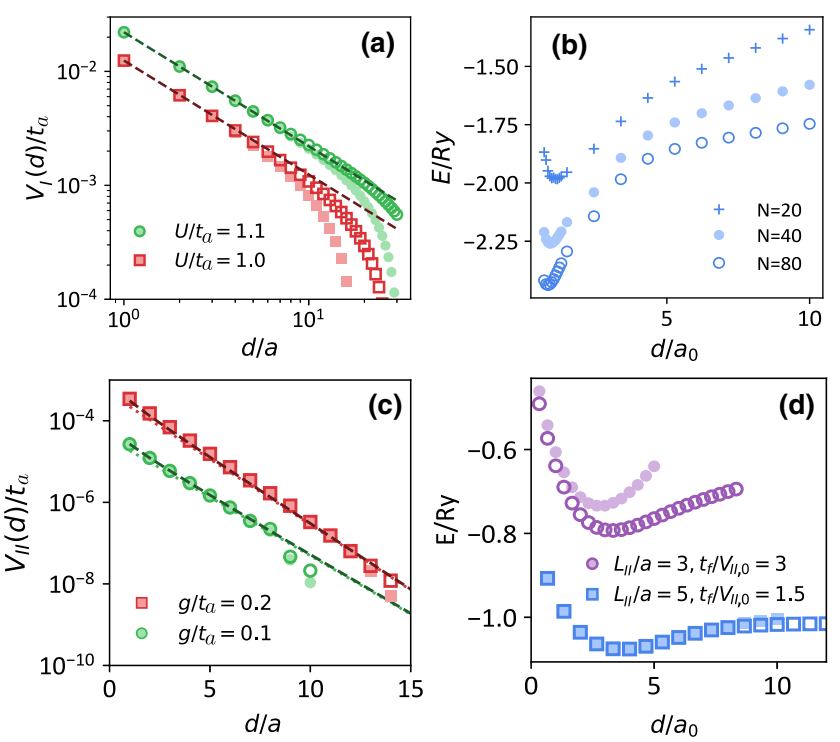

FIG. 4. (a) Energy of the single-boson bound state described by the first scheme Eq. (6) as the number of sites $d /$ a separating two fermions is modified. The dashed lines follow the scaling $V_{\mathrm{I}, 0} /(d / \mathrm{a})$. (b) Ground-state energy of the simulated Hamiltonian for $\mathrm{H}_{2}$ for different lattice sizes and the effective potential $V_{\mathrm{I}}(d)$. (c) Calculation of the repulsion mediated by the second scheme (8) between two fixed fermions separated $d /$ a sites (markers). The dashed line follows the analytical approximation (9). Edged markers corresponds to $N=80$ and colored ones to $N=40$. Here, $U=4.1 t_{a}$. (d) Molecular potential for a "pseudomolecule" of hydrogen, where both nuclear attraction and electronic repulsion follow the exponential scaling (9). Here, edged markers represent $N=60$, colored ones $N=30$, and $L_{\mathrm{II}} / \mathrm{a}=\left(2 \sqrt{\delta_{\mathrm{II}} / t_{a}}\right)^{-1}$. See Ref. [36] for details.

on-site repulsion $U$,

$$
H_{\text {med,I }}=-t_{a} \sum_{\langle\mathbf{i}, \mathbf{j}\rangle} a_{\mathbf{i}}^{\dagger} a_{\mathbf{j}}+U \sum_{\mathbf{i}} a_{\mathbf{i}}^{\dagger} a_{\mathbf{i}} f_{\mathbf{i}}^{\dagger} f_{\mathbf{i}},
$$

which translates into an effective repulsion between the fermions when the effect of the mediating atom is traced out,

$$
H_{\mathrm{ee}}=\sum_{\mathbf{i}, \mathbf{j}} V(|\mathbf{i}-\mathbf{j}|) f_{\mathbf{i}}^{\dagger} f_{\mathbf{i}} f_{\mathbf{j}}^{\dagger} f_{\mathbf{j}} .
$$

To obtain this expression, we assume we are in the regime in which the bosonic atom dynamics is faster than the movement of the fermions. In this first scheme, and for separations $d / \mathrm{a} \ll 0.06 e^{2 \pi t_{a} / U} \ll N$, this effective repulsion corresponds to, $V_{\mathrm{I}}(d) \approx V_{\mathrm{I}, 0} /(d / \mathrm{a})$, where $V_{\mathrm{I}, 0} \approx 6.4 e^{-2 \pi t_{a} / U} t_{a}$ (see SM [36]). This simple scheme then mediates an effective repulsion between the two fermionic atoms that scales as $1 / r$, matching the dependence of the distance of 3D molecular interactions, but now restricted to 2D [38]. We illustrate the dependence of this potential and its effect in the $2 \mathrm{D} \mathrm{H}_{2}$ molecule in Figs. 4(a) and 4(b), respectively. There, one can observe molecular potentials also for relatively small lattices and assess the error. The continuum limit is obtained in a similar regime as the $\mathrm{H}_{2}{ }^{+}$molecule case. 
Many-fermion models. By increasing the number of fermionic atoms in the lattice while maintaining a single mediating boson, one would see that not all interactions among pairs of fermions are equally weighted, precluding scalability. Intuitively, it is more favorable for the mediating atom to localize among the pair of fermions that are closer to each other, rather than in an equal superposition, so that not all interactions are equally considered. In Ref. [21], this challenge was overcome by including a cavity that symmetrizes these interactions. This cavity interaction is not available in the present, much simplified experimental setup, where interactions are mediated by a hopping atom instead of a spin excitation. Another option to induce a pairwise effective repulsion between these fermionic atoms would be Rydberg excitations that enable for long-range strong atomic interactions. In particular, one can induce dipole-dipole repulsive interactions that depend on their separation as $1 / d^{3}$ for distances smaller than the Rydberg blockade radius [39-42].

Here, instead, we present a second scheme that induces pairwise interactions by including as many mediating bosonic atoms as electrons need to be simulated. This proposal is scalable, at the price of modifying the scaling of the repulsive interaction [see Fig. 1(d)]. For these $N_{f}$ mediating atoms, we are going to consider two of their long-lived energy levels, that we call $a$ and $b$, separated by an energy shift $\Delta$. Level $b$ experiences an on-site repulsion $U$ when occupying the same site as a fermion, while the atoms in level $a$ live on a shallow lattice that allows them to move with tunneling rate $t_{a}$. Both levels are coupled through a Raman (or direct) transition of strength $g$. Besides, bosonic atoms in the $b$ level suffer an additional hard-core boson interaction $|W| \gg|U|$ which prevents doubly occupied states. The bosonic Hamiltonian then reads as

$$
\begin{aligned}
H_{\text {med,II }}= & -t_{b} \sum_{\langle\mathbf{i}, \mathbf{j}\rangle} b_{\mathbf{i}}^{\dagger} b_{\mathbf{j}}-t_{a} \sum_{\langle\mathbf{i}, \mathbf{j}\rangle} a_{\mathbf{i}}^{\dagger} a_{\mathbf{j}}+g \sum_{\mathbf{j}}\left(b_{\mathbf{j}}^{\dagger} a_{\mathbf{j}}+\text { H.c. }\right) \\
& +\Delta \sum_{\mathbf{j}} b_{\mathbf{j}}^{\dagger} b_{\mathbf{j}}+U \sum_{\mathbf{j}} b_{\mathbf{j}}^{\dagger} b_{\mathbf{j}} f_{\mathbf{j}}^{\dagger} f_{\mathbf{j}}+\frac{W}{2} \sum_{\mathbf{j}} b_{\mathbf{j}}^{\dagger} b_{\mathbf{j}}^{\dagger} b_{\mathbf{j}} b_{\mathbf{j}} .
\end{aligned}
$$

In particular, we are interested in the regime in which both levels are weakly coupled $g \ll \Delta$, and when the atomic states trapped in the $a$ lattice hop faster than in any of the other levels: $t_{a} \gg t_{b} \gg t_{F}$ [see Fig. 1(b)] [43]. This allows one to trace out the effect of the mediating atoms and write an effective Hamiltonian for the fermions. By using as many bosonic atoms as fermions, the hard-core boson interactions lead to a bound state in which all fermionic sites are equally occupied, getting a configuration in which the repulsion among each pair of atoms is equally weighted, as required by Eq. (6). For this configuration, the pairwise mediated interaction scales as

$$
V_{\mathrm{II}}(d) \approx V_{\mathrm{II}, 0} e^{-2 d \sqrt{\delta_{\mathrm{II}}} /\left(a \sqrt{t_{a}}\right)},
$$

for $d \sqrt{\delta_{\mathrm{II}}} /\left(a \sqrt{t_{a}}\right) \gg 1$, where $V_{\mathrm{II}, 0} \approx \frac{g^{4}}{8 \pi t_{a}^{2} \delta_{\mathrm{II}}}$, and $\delta_{\mathrm{II}}=U-$ $4 t_{a}+O\left(g^{2} / \Delta\right)$ (see Ref. [36]).

While this system differs from the molecular Hamiltonian observed in nature, it already captures the key features of the interactions appearing in molecular chemistry: nuclear attraction and electronic repulsion. It is then expected to reveal some of the features of chemical systems, including their electronic correlations. In Fig. 4(c), we show the effective repulsive potential induced by the second scheme for different values of detuning $\delta_{\text {II }}$, which is tunable and controls the characteristic length of the interaction. In Fig. 4(d), we illustrate the effect that this modified effective repulsion controlled by $\delta_{\text {II }}$ has two fermionic atoms hopping in the lattice, whose dependence on the distance is also mimicked by the tunable attractive nuclear interaction. This leads to a molecular potential of a "pseudomolecule" of hydrogen, where the bonding length and dissociation limit are observed.

Conclusions and outlook. To sum up, we have shown how ultracold atoms moving in 2D optical lattices can be used to simulate simplified models for quantum chemistry in today's experimental setups. We have observed that early experiments with a single simulating atom can pursue the timely goal of simulating the simplest discretized atom and molecule in this platform. In richer scenarios, bosonic atoms can mediate an effective repulsion between the simulated electrons, making repulsive interactions more experimentally accessible with state-of-the-art setups. Such simulators open up a number of possibilities for further research. First, they provide an experimental platform for which numerical methods used in quantum chemistry can be adapted and benchmarked. Lessons learned from these simulators could then be transferred back into improved algorithms for quantum chemistry. Second, one of the main challenges of these discretized 2D simulators is that their solutions approach the continuum result slower than in the 3D case. Fully characterizing this scaling may well lead to improved protocols that are less sensitive to the system size. Third, while this Rapid Communication provides strategies to engineer a pseudochemical Hamiltonian in ultracold atoms using bosonic atoms as a mediator, other platforms and strategies may also serve for this purpose. Identifying good candidates to simulate specific interactions in chemistry is a promising open field of research.

Acknowledgments. We acknowledge support from the ERC Advanced Grant QUENOCOBA under the EU Horizon 2020 program (Grant Agreement No. 742102). J.A.-L. acknowledges support from "la Caixa" Foundation (ID 100010434) through the fellowship No. LCF/BQ/ES18/11670016, the Spanish Ministry of Economy and Competitiveness through the "Severo Ochoa" program (SEV-2015-0522), Fundació Privada Cellex, Fundació Mir-Puig, and Generalitat de Catalunya through the CERCA program. A.G.-T. acknowledges support from the Spanish project No. PGC2018-094792-B-100 (MCIU/aEI/FEDER, EU) and from the CSIC Research Platform on Quantum Technologies PTI-001. T.S. acknowledges the Thousand-Youth-Talent Program of China and is supported by the NSFC No. 11974363. P.Z. acknowledges the EU Quantum Flagship PASQuanS. 
[1] T. Helgaker, P. Jorgensen, and J. Olsen, Molecular ElectronicStructure Theory (Wiley, Hoboken, NJ, 2014).

[2] P. Hohenberg and W. Kohn, Inhomogeneous electron gas, Phys. Rev. 136, B864 (1964).

[3] R. G. Parr and W. Yang, Density-Functional Theory of Atoms and Molecules (Oxford University Press, New York, 1989).

[4] A. C. Tsipis, DFT flavor of coordination chemistry, Coord. Chem. Rev. 272, 1 (2014).

[5] M. Head-Gordon, Quantum chemistry and molecular processes, J. Phys. Chem. 100, 13213 (1996).

[6] A. N. Alexandrova, A. I. Boldyrev, H. J. Zhai, and L. S. Wang, All-boron aromatic clusters as potential new inorganic ligands and building blocks in chemistry, Coord. Chem. Rev. 250, 2811 (2006).

[7] L. Domingo, M. Ríos-Gutiérrez, and P. Pérez, Applications of the conceptual density functional theory indices to organic chemistry reactivity, Molecules 21, 748 (2016).

[8] E. K.U. Gross and W. Kohn, Time-dependent density-functional theory, Adv. Quantum Chem. 21, 255 (1990).

[9] S. R. White, Density Matrix Formulation for Quantum Renormalization Groups, Phys. Rev. Lett. 69, 2863 (1992).

[10] M. Yang and S. R. White, Density-matrix-renormalizationgroup study of a one-dimensional diatomic molecule beyond the Born-Oppenheimer approximation, Phys. Rev. A 99, 022509 (2019).

[11] M. Motta, C. Genovese, F. Ma, Z.-H. Cui, R. Sawaya, G. K.-L. Chan, N. Chepiga, P. Helms, C. Jimenez-Hoyos, A. J. Millis, U. Ray, E. Ronca, H. Shi, S. Sorella, E. M. Stoudenmire, S. R. White, and S. Zhang, Ground-State Properties of the Hydrogen Chain: Dimerization, Insulator-to-Metal Transition, and Magnetic Phases, Phys. Rev. X 10, 031058 (2020).

[12] M. Motta, D. M. Ceperley, G. K.-L. Chan, J. A. Gomez, E. Gull, S. Guo, C. A. Jiménez-Hoyos, T. N. Lan, J. Li, F. Ma, A. J. Millis, N. V. Prokof'ev, U. Ray, G. E. Scuseria, S. Sorella, E. M. Stoudenmire, Q. Sun, I. S. Tupitsyn, S. R. White, D. Zgid, and S. Zhang, Towards the Solution of the Many-Electron Problem in Real Materials: Equation of State of the Hydrogen Chain with State-of-the-Art Many-Body Methods, Phys. Rev. X 7, 031059 (2017).

[13] M. Lubasch, J. I. Fuks, H. Appel, A. Rubio, J. I. Cirac, and M. C. Bañuls, Systematic construction of density functionals based on matrix product state computations, New J. Phys. 18, 083039 (2016).

[14] Y. Cao, J. Romero, J. P. Olson, M. Degroote, P. D. Johnson, M. Kieferová, I. D. Kivlichan, T. Menke, B. Peropadre, N. P. D. Sawaya, S. Sim, L. Veis, and A. Aspuru-Guzik, Quantum chemistry in the age of quantum computing, Chem. Rev. 119, 10856 (2019).

[15] A. Aspuru-Guzik, A. D. Dutoi, P. J. Love, and M. Head-Gordon, Simulated quantum computation of molecular energies, Science 309, 1704 (2005).

[16] R. Babbush, P. J. Love, and A. Aspuru-Guzik, Adiabatic quantum simulation of quantum chemistry, Sci. Rep. 4, 6603 (2013).

[17] B. P. Lanyon, J. D. Whitfield, G. G. Gillett, M. E. Goggin, M. P. Almeida, I. Kassal, J. D. Biamonte, M. Mohseni, B. J. Powell, M. Barbieri, A. Aspuru-Guzik, and A. G. White, Towards quantum chemistry on a quantum computer, Nat. Chem. 2, 106 (2010).
[18] I. Kassal, J. D. Whitfield, A. Perdomo-Ortiz, M.-H. Yung, and A. Aspuru-Guzik, Simulating chemistry using quantum computers, Annu. Rev. Phys. Chem. 62, 185 (2011).

[19] D. Wecker, M. B. Hastings, and M. Troyer, Progress towards practical quantum variational algorithms, Phys. Rev. A 92, 042303 (2015).

[20] O. Higgott, D. Wang, and S. Brierley, Variational quantum computation of excited states, Quantum 3, 156 (2019).

[21] J. Argüello-Luengo, A. González-Tudela, T. Shi, P. Zoller, and J. I. Cirac, Analogue quantum chemistry simulation, Nature (London) 574, 215 (2019).

[22] I. Bloch, J. Dalibard, and W. Zwerger, Many-body physics with ultracold gases, Rev. Mod. Phys. 80, 885 (2008).

[23] T. Esslinger, Fermi-Hubbard physics with atoms in an optical lattice, Annu. Rev. Condens. Matter Phys. 1, 129 (2010).

[24] C. Gross and I. Bloch, Quantum simulations with ultracold atoms in optical lattices. Science 357, 995 (2017).

[25] We acknowledge that other analog simulators based on fermionic atoms trapped in optical lattices have been proposed to emulate the molecular potentials of benzenelike molecules [47] or simulate ultrafast dynamics in strong fields $[48,49]$. In contrast to them, Ref. [21] and the present proposal allow one to go beyond the local interactions naturally found in cold atoms, simulating the nonlocal fermionic repulsion that appears in typical quantum chemistry problems.

[26] M. J. O'Rourke, Z. Li, and G. K.-L. Chan, Efficient representation of long-range interactions in tensor network algorithms, Phys. Rev. B 98, 205127 (2018).

[27] Z. Li, M. J. O'Rourke, and G. K.-L. Chan, Generalization of the exponential basis for tensor network representations of longrange interactions in two and three dimensions, Phys. Rev. B 100, 155121 (2019).

[28] Throughout the text, bold variables denote $2 \mathrm{D}$ vectors.

[29] In order to prevent the divergence in the origin, positions $r_{n}$ of the nuclei are shifted half a site from the lattice nodes in the $y$ direction.

[30] Considering that the electronic dynamics is much faster than the nuclear one, their equations can be decoupled (Born-Oppenheimer approximation). The position $\left\{\tilde{\boldsymbol{r}}_{n}\right\}_{i=n \ldots N_{n}}$ of the $N_{n}$ nuclei is considered fixed during the calculation of the electronic Hamiltonian $H_{\text {cont }}$, for the $N_{f}$ electrons in positions $\left\{\boldsymbol{r}_{i}\right\}_{i=1 \ldots N_{f}}, \quad H_{\text {cont }}=-\sum_{i=1}^{N_{f}} \frac{\hbar^{2}}{2 m_{e}} \nabla_{i}^{2}-$ $\sum_{i=1}^{N_{f}} \sum_{n=1}^{N_{n}} Z_{n} V\left(\left|\boldsymbol{r}_{i}-\tilde{\boldsymbol{r}}_{n}\right|\right)+\sum_{i \neq j=1}^{N_{f}} V\left(\mid \boldsymbol{r}_{i}-\boldsymbol{r}_{j} \|\right)$, where $m_{e}$ is the mass of the electron and $Z_{n}$ is the atomic number of nucleus $n$. The first term then describes the kinetic energy of the electrons, the second its nuclear attraction following the potential $V(r)$, and the third the electronic repulsion.

[31] This externally induced potential could eventually mimic the effect of inner-shell electrons as well.

[32] J.-y. Choi, S. Hild, J. Zeiher, P. Schauß, A. Rubio-Abadal, T. Yefsah, V. Khemani, D. A. Huse, I. Bloch, and C. Gross, Exploring the many-body localization transition in two dimensions, Science 352, 1547 (2016).

[33] B. Zaslow and M. E. Zandler, Two-dimensional analog to the hydrogen atom exact analytical solutions of a two-dimensional hydrogen atom in a constant magnetic field, Am. J. Phys. 35, 1118 (1967). 
[34] J.-L. Zhu and J.-J. Xiong, Hydrogen molecular ions in two dimensions, Phys. Rev. B 41, 12274 (1990).

[35] As compared to the three-dimensional case, $R y(2 D)=$ $4 \mathrm{Ry}(3 \mathrm{D})$, and $2 \mathrm{a}_{0}(2 \mathrm{D})=\mathrm{a}_{0}(3 \mathrm{D})$. Throughout the text, we will omit the (2D) labeling.

[36] See Supplemental Material at http://link.aps.org/supplemental/ 10.1103/PhysRevResearch.2.042013 for a discussion of the scaling of the spectrum of the discretized 2D Hamiltonian as the lattice size increases; a derivation of the effective interaction mediated by a single boson with one long-lived state; a discussion on the effective interaction mediated by several mediating atoms with two long-lived internal states; and further details about the numerical methods used to obtain Figs. 2-4, which includes Refs. [21,44-46].

[37] S. H. Patil, Hydrogen molecular ion and molecule in two dimensions, J. Chem. Phys. 118, 2197 (2003).

[38] Note that this choice of nuclear potential differs from the one encountered in a flatland world, in which Coulomb's law leads to interactions that scale as $\propto \log (r)$.

[39] M. D. Lukin, M. Fleischhauer, R. Cote, L. M. Duan, D. Jaksch, J. I. Cirac, and P. Zoller, Dipole Blockade and Quantum Information Processing in Mesoscopic Atomic Ensembles, Phys. Rev. Lett. 87, 037901 (2001).

[40] S. Ravets, H. Labuhn, D. Barredo, L. Béguin, T. Lahaye, and A. Browaeys, Coherent dipole-dipole coupling between two single Rydberg atoms at an electrically-tuned Förster resonance, Nat. Phys. 10, 914 (2014).
[41] M. Saffman, T. G. Walker, and K. Mølmer, Quantum information with Rydberg atoms, Rev. Mod. Phys. 82, 2313 (2010).

[42] However, the bare interaction is anisotropic in nature.

[43] A. Heinz, A. J. Park, N. Šantić, J. Trautmann, S. G. Porsev, M. S. Safronova, I. Bloch, and S. Blatt, State-Dependent Optical Lattices for the Strontium Optical Qubit, Phys. Rev. Lett. 124, 203201 (2020).

[44] S. Katsura and S. Inawashiro, Lattice Green's functions for the rectangular and the square lattices at arbitrary points, J. Math. Phys. 12, 1622 (1971).

[45] M. Abramowitz and I. A. Stegun, Handbook of Mathematical Functions with Formulas, Graphs and Mathematical Tables, 9th ed. (Dover, New York, 1972).

[46] S. Schmid, G. Thalhammer, K. Winkler, F. Lang, and J. H. Denschlag, Long distance transport of ultracold atoms using a 1D optical lattice, New J. Phys. 8, 159 (2006).

[47] D.-S. Lühmann, C. Weitenberg, and K. Sengstock, Emulating Molecular Orbitals and Electronic Dynamics with Ultracold Atoms, Phys. Rev. X 5, 031016 (2015).

[48] S. Sala, J. Förster, and A. Saenz, Ultracold-atom quantum simulator for attosecond science, Phys. Rev. A 95, 011403(R) (2017).

[49] R. Senaratne, S. V. Rajagopal, T. Shimasaki, P. E. Dotti, K. M. Fujiwara, K. Singh, Z. A. Geiger, and D. M. Weld, Quantum simulation of ultrafast dynamics using trapped ultracold atoms, Nat. Commun. 9, 2065 (2018). 